Original Article

\title{
Impact of patients' judgment skills on asthma self-management: a pilot study
}

\author{
Ana Maria Moreno Londoño, Peter J. Schulz \\ Institute of Communication and Health, University of Lugano, Switzerland
}

Significance for public health

Patients' health literacy has a great impact on their health behaviours and their health outcomes. Therefore, it has become more and more common to measure health literacy within the healthcare setting to determine the most effective approach to target patients. The measurement of asthma judgment skills contribute to a deeper understanding of patients' asthma self-management in crucial topics for asthma control, and have the advantage of assessing the specific abilities needed for this particular condition, which in turn benefits the translation of the findings from the use of this tool into strategies that directly tackle the needs of asthma patients.

\section{Abstract}

Background. The majority of current health literacy tools assess functional skills including reading, writing, and numeracy. Although these tools have been able to underline the impact of such skills on individuals' health behaviour, there is a need for comprehensive measures to examine more advanced skills. The individual's ability to use health-related information considering his/her own health context, and judging positive and negative consequences of their decisions has been conceptualized as judgment skills. The present study used a newly developed judgment skills tool to explore asthma selfmanagement practices.

Design and methods. Eighty asthma patients were recruited from medical offices during the year 2013. The questionnaire was selfadministered and contained health literacy questions, the judgment skill tool, the Asthma Control Test, and several self-management questions.

Results. Sixty-nine percent of participants had adequate health literacy, while $24 \%$ and $5 \%$ had marginal and inadequate levels, respectively. The high-judgment group referred more to their doctor when experiencing asthma problems $t(76)=-2.18, \mathrm{P}<0.032$; complied more with the use of their control medicine $t(77)=-3.24, \mathrm{P}<0.002$ and went more regularly to the doctor $t(78)=-1.80, \mathrm{P}<0.038$ (one-tailed) than the low-judgment group.

Conclusions. The judgment skills tool can help identify asthma patients' health information use and reveal how this use may affect some self-management practices.

\section{Introduction}

In the last decades, enormous attention has been paid to health literacy due to its influence on health behaviours and health outcomes and there is a robust body of literature focusing on this relationship. ${ }^{1-4}$
However, two main aspects are still discussed by the research community for this concept to be fully considered as an effective approach. One concerns its conceptualization, as to date there is not a unique definition for it, and the other one its measurement. ${ }^{5}$ The most commonly used measures have focused on assessing reading, writing, and numeracy skills. ${ }^{6}$ Although so far the tools measuring these functional skills have shown a well-established relationship between health literacy and health outcomes, ${ }^{3}$ these tools fail to capture more advanced health literacy skills needed by individuals to function properly within a health care context, ${ }^{7}$ including decision-making, analytical thinking, pondering abilities, information use, informatics and communicative skills. These advanced skills among others allow patients to be autonomous in navigating the health care system, participate actively in their own and their families' health care, make informed decisions, and collaborate efficiently with healthcare professionals. All this points towards the need to develop reliable tools that can assess skills that go beyond these functional abilities. Different attempts have been carried out to develop other measures able to capture more advanced health literacy skills. Chew et al. ${ }^{8}$ for instance developed a screening tool to assess individuals' understanding of health material and the use of this; Ishikawa et al. ${ }^{9}$ went further and developed a screening tool assessing different dimensions of health literacy such as individuals' capabilities to extract health information, derive meaning from it (communicative literacy), and critically use it (critical literacy). Despite these noticeable efforts to advance health literacy measurement, findings from other studies using these tools are inconclusive regarding their capacity to measure more advanced health literacy dimensions. ${ }^{10,11}$ Another recent approach that fits into the conceptualization of advanced health literacy skills has been proposed by Schulz and Nakamoto. ${ }^{12}$ One of the dimensions of the authors' theoretical framework, known as judgment skills, focuses on the individuals' abilities to adapt and apply health information according to the health context. These skills allow the individual to subtract and generalize information, to build knowledge that can be applied differently according to the situation. ${ }^{12}$ Thus, it has been hypothesized that individual with higher judgment skills are able to respond better to a particular health situation. The conceptualization of judgment skills has been recently and successfully operationalized in a scenario based-tool for the context of asthma self-management. ${ }^{13}$

The tool was developed in the asthma context because this chronic condition poses high demands on patients' self-care routines. Patients need to follow strict medical regimens, use medicines properly, avoid asthma triggers, and recognize symptoms. However, if asthma health information is not properly understood and integrated by patients, a proper asthma control is difficult to achieve, which is at the end the final purpose for managing this condition. Therefore, the characteristics present in asthma self-management make it an appropriate condition to develop and test the proposed judgment skills. In addition to this, it has been pointed out by other authors that health literacy skills should involve more content-specific skills and health-related knowledge that depends on the health condition. ${ }^{7}$ Thus it is crucial to devel- 
op content-specific tools as the knowledge and skills required vary.

The purpose of the present work is to continue the evaluation of this newly developed tool and find out whether patients with higher judgment skills perform better than patients with low judgment skills in different asthma self-management practices such as medicine usage, trigger control, symptoms recognition, information seeking, doctorpatient communication, and exercise. Furthermore, this study aimed to investigate the associations between patients' past self-management experiences and their impact on responding to similar situations.

\section{Design and methods}

A cross-sectional survey was conducted among 80 asthma patients recruited from medical offices in the Italian-speaking region of Switzerland using a self-reported paper-pencil questionnaire in Italian. It contained questions regarding functional health literacy, judgment skills, past self-management experiences, the Asthma Control Test (ACT), asthma self-management, and demographics.

\section{Measures}

Different validated scales were assembled into a questionnaire to explore the study variables. The following is a description of the variables and the scales used to measure them:

Functional health literacy. This was assessed by a validated scale that included three screening questions: ${ }^{8}$ How often do you have someone help you read hospital materials?; How often do you have problems learning about your medical condition because of difficulty understanding written information?; How confident are you filling out forms by yourself?. These were measured on a 5 -point scale ranging from always to never (score 1-5).

Judgment skills. These were evaluated by the scenario-based tool developed in a previous study. ${ }^{13}$ It included nineteen scenarios with four response option each, describing self-management situations regarding exercise, doctor-patient communication, information seeking, triggers control, symptoms recognition, and medicine use. Every response option was transformed into a score ranging from 1-4 (most inadequate to most adequate). The ranking of the response options and the validity of the scenarios was determined in a prior study by a Delphi panel (i.e. panel of experts gathered to achieve consensus about a particular topic) composed of twelve physicians in the field of lung diseases. $^{13}$

Past experience. Following each scenario a yes/no question was asked about prior experience on a similar situation as the one described in the scenario. The purpose of this was to verify whether the described scenarios matched the most common situations for asthma patients and whether past experience has an association with the response given to the scenario.

Test for asthma control (ACT). This validated and widely use test included five questions measured in a 5-point scale. They assessed asthma symptoms and medicine usage in the last four weeks, ${ }^{14}$ e.g., During the past 4 weeks, how often have you had shortness of breath?

Asthma self-management. A pull of seven questions measured patients' care practices; some were drawn from a study on asthma management, ${ }^{15}$ and others from asthma guidelines, ${ }^{16}$ the scale ranged from always to never ( 3 and 5 point scales) and included questions such as I take my rescue medicine as indicated by my doctor. These questions were used as outcome measures.

Demographics. These were included at the end of the questionnaire with the aim of describing the study population.

The questionnaire was pre-tested among ten asthma patients who did not belong to the pilot study, and it was revised by two experts in questionnaire development before conducting the study.
Ethical approval was granted from the ethical committee of the Canton Ticino, Italian-speaking region of Switzerland (i.e. Comitato Etico Cantolane FN132445.Rif.CE2453).

\section{Recruitment}

Thirty one out of sixty-six health care professionals, working in the field of asthma, including pulmonologists, allergists, general practitioners and physiotherapists, in the Italian part of Switzerland accepted the invitation to participate in the recruitment of patients. Generally, non- participation was due to the lack of asthma patients. The recruitment of the patients was done during the medical consultation or while waiting for the appointment by the medical assistants. Asthma patients were offered to fill out the questionnaire anonymously and at their convenience, either in the waiting room or at home, to encourage participation. The majority of participants chose the second option, for which a stamped return envelope was provided as well. Besides the instructions on how to fill out the questionnaire, patients received a leaflet containing an informed consent form, the approval of the ethics committee, general information about the project, and the funding source.

\section{Participants}

The eligibility criteria for patients to participate were to be at least 18 years old, being diagnosed with asthma by a physician, being on treatment or having had asthma symptoms or attacks in the previous year, read fluent Italian and live in the Italian-speaking region of Switzerland. Based on these criteria patients were recruited in approximately six months. The majority of the respondents were females, $67 \%$ (54), with a mean age of 46 years $(\mathrm{SD}=15)$; participants' ages ranged from 18 to 80 . Educational attainment was categorized into three groups: primary and secondary school 19\% (15), high schooVapprenticeship $61 \%$ (49), and university $19 \%$ (15). More than half of the study population presented adequate health literacy $69 \%$ (55), while $24 \%$ (19) and 5\% (4) had marginal and inadequate levels, respectively. Moreover, the mean of years suffering from asthma was $21(\mathrm{SD}=14)$, with $52 \%$ (42) suffering from persistent asthma and $44 \%$ (35) from intermittent asthma, and there was a high rate of non-smokers $82 \%$ (66). According to the ACT, most of the population had their asthma under control in the last four weeks $59 \%$ (47), and the majority of participants were using asthma medicines at the time of the survey $72 \%$ (58).

\section{Statistical analysis}

Descriptive statistics were used to define the study population. For some analysis missing values were replaced by individual scale means, when at least $50 \%$ of the scale items were filled in, to guarantee representative results. Kendall's tau_b and Pearson correlations were used when appropriate, according to the continuous or nominal nature of the variables. Correlations were used to explore possible relationships among self-management behaviours, judgment skills, health literacy, education attainment and asthma control. A composite score of judgment skills was computed for every participant based on the final eleven scenarios (i.e. eight scenarios were deleted during preliminary analysis, due to their low performance, including poor discrimination) ranging theoretically from 11 to 44, with higher values representing better judgment skills. A Low and a High judgment group were formed by splitting the score along the median (Low: 28-36, High 37-44). This partition is supported by the data since $38 \%$ of people in the Low judgment group selected the most or second most inadequate option four times on average, while $47 \%$ of participants in the High judgment group selected the same options only once on average. This indicates, indeed, that the second group had better judgment skills on responding to the described situation on the scenarios.

Furthermore, several independent t-tests were carried out to explore 
possible group differences between the Low and the High judgment groups on self-management behaviours. P-values $<0.05$ were considered statistically significant.

\section{Results}

This section is presented in several subsections. The first part reports on the main findings of the study regarding the impact of judgment skills on asthma self-management, followed by a description of the effect of participants' past experiences on the response to the situations, and it continues reporting the judgment skills tools' performance. The last section shows the correlations found among the different studied variables including functional health literacy, education, ACT, etc.

\section{Impact of judgment skills on asthma self-management}

Findings from the judgment skills tool suggest that only $29 \%$ of participants in the survey will give away the pet as suggested by the doctor when indicated as a major trigger of asthma (sc. 7). More than half of the participants (67\%) will use the rescue medicine in public places to alleviate the experienced symptoms (sc. 9). Half of the participants will discuss with their doctor concerns about using a medicine known for their side effects (sc. 2). $65 \%$ will discuss with their physician possible side effects found on Internet of a prescribed medicine (sc. 5). Only $60 \%$ of patients will use rescue medicine to alleviate symptoms and continue to take the preventive medicine as indicated by the doctor (sc. 10). Fifty nine percent of participants thought that their asthma was controlled even after experiencing shortening of breath, coughing, waking up at night, and not being able to do routine activities for a while (sc. 8), which from a medical point of view suggest an uncontrolled asthma. ${ }^{10}$

Participants in the High-judgment group recurred more to their doctor when experiencing problems with their asthma $(M=3.86, \mathrm{SD}=1.31)$ than their counterparts in the Low-judgment group $(M=3.24$, $\mathrm{SD}=1.21$ ), $t(76)=-2.18, \mathrm{P}<0.032$ (Figure $1 \mathrm{~A}$ ).

Similarly, the High-judgment group was more compliant with the use of their control medicine $(M=4.29, \mathrm{SD}=0.99)$, in terms of interrupting it less than the Low-judgment group $(M=3.41, \mathrm{SD}=1.33), t(77)=-3.24$, $\mathrm{P}<.002$ (Figure 1B). Moreover, people with higher judgment skills went more regularly to the doctor $(M=4.47, \mathrm{SD}=0.81)$ than participants in the Low judgment group $(M=4.07, \mathrm{SD}=1.13 t(78)=-1.80, \mathrm{P}<0.038$ (One-tailed) (Figure 1C).

\section{How do past experiences affect asthma self-manage- ment}

In the questionnaire, each of the scenarios was followed by a question on having experienced a similar situation in the past. Positive responses ranged from $17 \%$ (sc. 5) to $72 \%$ (sc. 10). The topics of the scenarios more commonly experienced by participants were: experiencing symptoms in a public place (60\% sc. 9), perceiving asthma symptoms (61\%, sc. 8), fixing regular visits to the doctor $(61 \%$, sc. 4$)$, and using the control medicine (67\%, sc. 11).

Having experienced a similar situation as described in the scenarios was related in some cases with self-management behaviours. People who in the past experienced symptoms while exercising $(M=3.56$, $\mathrm{SD}=1.24)$ interrupted their control medicine more often than the ones who had never experienced a similar situation $(M=4.12, \mathrm{SD}=1.25)$, $t(77)=-1.99, \mathrm{P}<0.050$. In the same way people who experienced asthma symptoms in a public place went more often to the doctor when experiencing problems with their asthma $(M=3.79, \mathrm{SD}=1.178)$ than people who had never been in a similar situation $(M=3.13, \mathrm{SD}=1.360)$, $t(76)=2.270, \mathrm{P}<0.026$. Furthermore, people who had experienced symptoms in public places had less control over their asthma $(M=3.64$, $\mathrm{SD}=0.913)$ than people who had never had a similar situation $(M=4.11$, $\mathrm{SD}=0.854), t(77)=-2.305, \mathrm{P}<0.024$.

\section{Performance of the judgment skill tool}

The initial version of the instrument included 19 scenarios covering the topics of exercise and medicine usage, doctor-patient communication, information seeking, triggers control, symptoms recognition, and medicine usage. After preliminary analysis, eight scenarios were deleted for different reasons. A scenario was deleted when more than $5 \%$ of the responses were missing. This occurred in a scenario that did not concern the whole study population, such as smoking behaviour. Scenarios were also deleted when the majority of the participants selected the best answer, indicating lack of discrimination of responses, and when there was a low percentage of participants having experienced a similar situation in the past. Despite these deletions the initial six self-management topics remained represented in the eleven final scenarios (Appendix).

\section{Correlations}

From the section on self-management three variables significantly

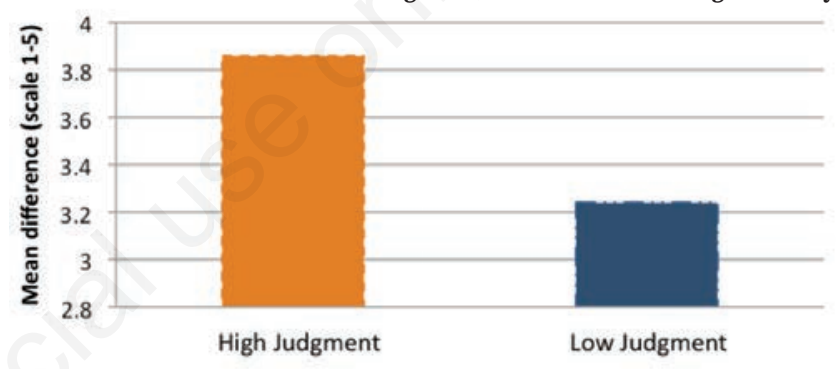

A Judgment Skills by Group

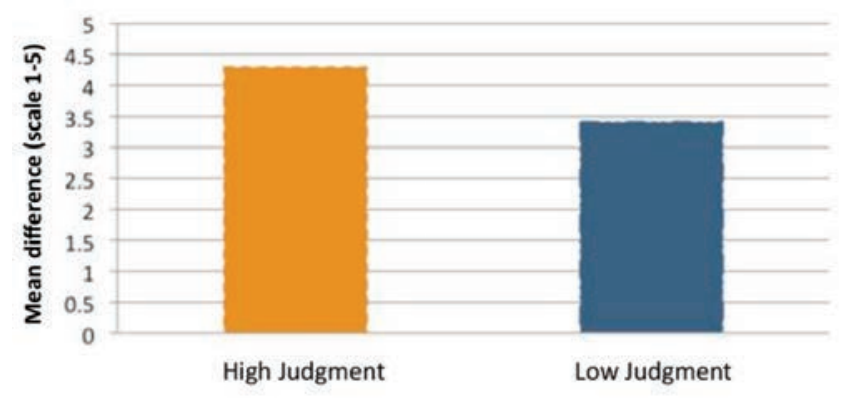

B

Judgment Skills by Group

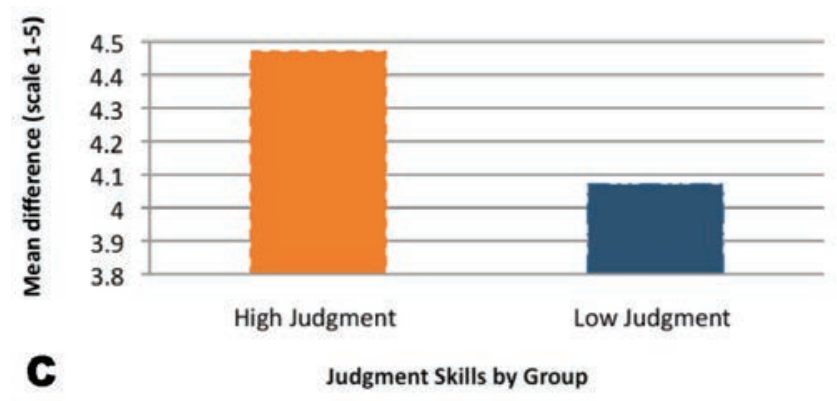

Figure 1. A) Patients going to the doctor when experiencing problems with their asthma. B) Control medicine compliance. C) Regular visits to the physician. 
Table 1. Correlations among health literacy, judgment skills, Asthma Control Test, and asthma self-management.

\begin{tabular}{|c|c|c|c|c|c|c|c|c|c|c|}
\hline $\begin{array}{l}\text { Health } \\
\text { literacy }\end{array}$ & $\begin{array}{l}\text { Judgment } \\
\text { score }\end{array}$ & $\begin{array}{l}\text { Educational } \\
\text { attainment }\end{array}$ & $\begin{array}{l}\text { Regular } \\
\text { visits } \\
\text { to docs }\end{array}$ & $\begin{array}{c}\text { Rescue } \\
\text { medicine } \\
\text { use }\end{array}$ & $\begin{array}{c}\text { Control } \\
\text { medicine } \\
\text { use }\end{array}$ & $\begin{array}{l}\text { Interrupt } \\
\text { control } \\
\text { medicine }\end{array}$ & $\begin{array}{c}\text { Avoidance } \\
\text { of } \\
\text { triggers }\end{array}$ & $\begin{array}{l}\text { Prescriptior } \\
\text { refill }\end{array}$ & $\begin{array}{l}\text { Call doctor } \\
\text { if problems }\end{array}$ & $\mathrm{ACT}$ \\
\hline
\end{tabular}

Health literacy

\begin{tabular}{lcccccccccc} 
Judgment score & 0.08 & - & & & & & & & & \\
Educational attainment & 0.17 & 0.09 & - & & & & & & \\
Regular visits to docs & -0.12 & $0.22^{*}$ & -0.07 & - & & & & & \\
\hline Rescue medicine use & -0.01 & 0.15 & -0.18 & 0.13 & - & & & & \\
Control medicine use & 0.05 & 0.12 & -0.14 & $0.29^{* *}$ & $0.30^{* *}$ & - & & & \\
\hline Interruption of control & -0.12 & $0.37^{* *}$ & $-0.20^{*}$ & $0.42^{* *}$ & $0.36^{* *}$ & $0.53^{* *}$ & - & & - \\
Avoidance of triggers & $-0.28^{*}$ & 0.11 & 0.02 & $0.49^{* *}$ & 0.06 & 0.01 & 0.18 & - & \\
\hline Prescription refill & 0.03 & 0.21 & -0.04 & $0.31^{* *}$ & 0.02 & 0.16 & 0.20 & $0.24^{*}$ & - \\
Call doctor if problems & -0.14 & $0.25^{*}$ & 0.00 & $0.31^{* *}$ & 0.17 & 0.05 & $0.27^{*}$ & 0.17 & 0.22 & - \\
\hline ACT & 0.12 & 0.13 & -0.01 & 0.05 & 0.06 & 0.10 & 0.03 & -0.17 & 0.08 & -0.21 \\
\hline
\end{tabular}

*Correlation is significant at the 0.05 level (2-tailed). ${ }^{* *}$ Correlation is significant at the 0.01 level (2-tailed).

correlated with judgment skills: fixing regular appointments with the physician, interrupting the use of control medicine, and addressing the physician if experiencing problems with their asthma. Furthermore selfmanagement practices, education, health literacy, and the ACT showed other significant correlations (Table 1).

The three items measuring functional health literacy were correlated independently with educational attainment. Findings indicated that the question: How often do you have someone help you read hospital materials? was significantly correlated with the level of participants' education $0.23(\mathrm{P}<0.05)$, indicating that not having problems to read hospitals materials is associated with having a higher educational attainment.

\section{Discussion and Conclusions}

The purpose of the present work was to continue the evaluation of a newly developed judgment skills tool and find out whether patients with higher judgment skills perform better than patients with low judgment skills in different asthma self-management practices such as medicine usage, trigger control, symptoms recognition, information seeking, doctor-patient communication, and exercise. Furthermore, this study aimed to investigate the associations between patients' past self-management experiences and their impact on responding to similar situations. Findings from this study showed that patients with higher judgment skills recurred more often to the doctor when they experienced problems with their asthma, were more compliant with the use of their control medicine, and made appointments with their physicians more regularly than patients with low judgment skills. Moreover, significant correlations were found between several self-management practices and participants' past self-management experiences. Thus, people experiencing asthma symptoms while exercising (sc. 1) were more prone to interrupt the use of their control medicine. Likewise, individuals who had experienced asthma symptoms in public (sc. 9) tended to go more often to the doctor and had less control over their asthma than people who did not had similar experiences in the past.

These findings support the recognized key role of patients in the self-management of asthma. ${ }^{17-19}$ In general, asthma can be considered as a major impediment when managed poorly, or as a minor inconvenience when managed effectively. ${ }^{20}$ An appropriate self-management includes the use of medicines timely and regularly, and recognition of asthma symptoms. When self-management is not carried out effectively, symptoms and asthma attacks arise, leading patients to experience a decrease of quality of life, ${ }^{21}$ increased hospitalizations, ${ }^{22}$ unscheduled doctor's and, emergency department's visits, and the days lost of work/school. ${ }^{17}$ Due to the importance of asthma self-management, every patient should possess the skills to carry out activities and behaviours that lead him/her to an appropriate self-care. Judgment skills allow individuals to built knowledge collected from past experiences, and from other sources of information (e.g. doctors, internet, etc) and then use it according to the health situation encountered. For instance an asthma patient when experiencing symptoms while exercising could respond to the situation by using his medicine on time and stopping the activity. Such a response is partly a result of the appropriate use of health information built upon past experiences, evaluation of pros and cons of action taken, and communication with doctors, which are in sum judgment skills.

Health literacy has been considered for the past decades as a powerful factor influencing individuals' health behaviour. To date, the most widely used measures of health literacy focus on assessing reading, writing and numeracy skills. ${ }^{6}$ Although so far these measures have shown a well-established relationship between health literacy and health outcomes, ${ }^{3}$ they fail to capture more advanced health literacy dimensions such as an individual's health information use. ${ }^{7}$ Judgment skills were conceptualized within the health literacy context in the Health Empowerment Model. ${ }^{12}$ These skills allow patients to exert a greater control over their health condition, enabling them to actively participate in their self-care. Assessing judgment skills, especially in the context of chronic diseases where patients have a great deal of responsibility is important in order to understand to what extent the presence or absence of these skills influence health behaviours and health outcomes of patients.

Several authors have highlighted a latent dissonance between what it is believed to be known by patients and what is really known. ${ }^{23} \mathrm{~A}$ study on asthma self-management found worrying differences between practical self-management knowledge and behaviours during acute attacks. In most of the cases knowledge exceeded the reported action taken. ${ }^{24}$ Numerous educational programs rely heavily on information transfer as opposed to teaching skills to patients. However, knowing a range of facts about a disease does neither guarantee a change in behaviour, ${ }^{25}$ nor a proper use of information. A study addressing the influence of knowledge and behaviour on asthma self-management concluded that while the acquisition of practical asthma knowledge is 
an important part of asthma self-management, it is not sufficient for a satisfactory asthma self-management behaviour. ${ }^{24}$ Similarly, another study concluded that educational programs improved knowledge but did not reduce asthma morbidity. ${ }^{26}$ What these results suggest is that measuring only knowledge will not lead to a clear understanding of how it is being applied. The judgment skills tool can contribute to overcome this gap. Asthma patients are expected to play an autonomous role in self-management. They are responsible for recognizing symptoms, adjusting medicines, avoiding triggers, and being able to communicate problems and concerns to their health care providers, among others. All these competencies require skilful patients able to use and apply information according to the situation. Thus, they can achieve an adequate self-management that leads to positive health outcomes such as better asthma control and improved quality of life. Results from this study show that the High-judgment group recurred more to the doctor when problems arise and was more compliant with the use of the control medicine. Both self-management behaviours go in line with what is expected of an appropriate self-care. ${ }^{16} \mathrm{~A}$ lack of appropriate response by patients experiencing an onset of an asthma attack (i.e. not calling emergency services) has been linked to inappropriate practical knowledge. ${ }^{24}$ Likewise, a study conducted among African American adolescents regarding asthma self-management found that $23 \%$ of the participants never sought help from other people at the first signs of breathing problems, and only $49 \%$ reported stopping their activities when experiencing breathing problems. ${ }^{27}$ Furthermore, several studies have reported that some of the reasons for under-using medicine are due to the misunderstanding of therapy by patients, poor knowledge on asthma medication, ${ }^{28}$ and fears and misconceptions about side-effects. ${ }^{29}$ Results from a study of asthma self-management with adolescents reported that non-compliance with the therapy was partly because patients believed that the medical regimen did not have any effect on their symptoms, hence patients selected which aspects of the regimen to follow, departing from medical guidance. ${ }^{30}$

Bender et $a l .{ }^{31}$ found that adherence to a daily controller medication was generally below the prescribed level, despite the fact that many participants suffered from severe asthma and frequent symptoms. Patients with higher judgment skills in this study reported better compliance with this medication. This might indicate a better communication with health care providers. Several studies have shown that, if there is a good partnership between doctor and patient, it increases adherence to the treatment, recall, and understanding of medical information. ${ }^{32}$

Among the limitations of this study is the reliance on the use of a self-reported tool, with neither objective measures of lung functioning to assess the level of asthma control, nor objective health behaviour measurements that indicate that patients behave as reported. ${ }^{33}$ The advantages of self-administered tools are that discomfort or embarrassment are avoided. Although the scenarios describe common situations for asthma patients, the amount of reading might pose a burden on people with poor reading skills, discouraging participants to participate in the study and imposing a risk for random responses. To lessen those risks participants were given the time to fill in the questionnaire at home. Furthermore, this study acknowledges the possible loss of measurement information due to dichotomization of the variables using the median split. However, this partition was necessary due to skewness of the composite score of judgment skills, with a high tendency of selecting the best options. However, for this particular case the median split has been accepted by other authors, ${ }^{34}$ and the data itself showed a difference between the split groups. Since the tool is context-bound it would require more effort to translate it into another context. To the best of our knowledge, health information use, expressed as judgment skills, within the asthma context has not been explored before. Our findings contribute to the health literacy field by providing an assessment tool that goes beyond the functional skills shedding light on how limited health information use leads to adverse self-management behaviours. Judgment skills play an important role on the health behaviour of asthma self-management. The use of this tool is recommended to identify self-care aspects that should be addressed in patient education.

Correspondence: Ana Maria Moreno Londoño, Institute of Communication and Health, University of Lugano, Via Giuseppe Buffi 13, CH-6904 Lugano, Switzerland.

Tel.: +41.078.766.1816.

E-mail: anamorenol@gmail.com

Key words: health literacy, judgment skills, asthma self-management, pilot study.

Acknowledgements: the authors thankfully acknowledge all physicians, physiotherapist, and The Lega Polmonare Ticinese for having collaborated with the recruitment of patients. Special thanks to Dr. Gianfranco Bolognini, Dr. Adriano Martinelli, Dr. Antonio Satta, Dr. Andrea Azzola, Dr. Gianluca Vanini, Dr. Gianluca Calderari, Dr. Massimiliano Fontana, Dr. Guido Domenighetti, Dr. Jean Pierre Lantin, Dr. Sándor Györik, Dr. Bruno Naccini, Dr. Franco Quadri, Dr. Paola Bettelini Lurà, Dr. Andreas Blatter, Dr. Claudia Borelli, Marco Massimo Calì, Roberto Bianchini, and Alessandra Bianchini. Moreover, we express our gratitude to all patients and assistants that participated in this study. The Swiss National Sciences Foundation financially supported this study.

Contributions: the authors contributed equally.

Conflict of interests: the authors declare no potential conflict of interests

Received for publication:15 July 2014.

Accepted for publication: 6 November 2014.

(C) Copyright A.M.M. Londoño and P.J. Schulz, 2014

Licensee PAGEPress, Italy

Journal of Public Health Research 2014; 3:307

doi:10.4081/jphr.2014.

This work is licensed under a Creative Commons Attribution NonCommercial 3.0 License (CC BY-NC 3.0).

\section{References}

1. WHO. The solid facts: health literacy. Geneve: World Health Organization. 2013. Available from: http://www.euro.who.int/ _data/assets/pdf_file/0008/190655/e96854.pdf

2. Institute of Medicine. Health literacy: a prescription to end confusion. Washington, DC: National Academies Press. 2004.

3. Agency for Healthcare Research and Quality. Health literacy interventions and outcomes: an update of the literacy and health outcomes systematic review of the literature. Available from: http://effectivehealthcare.ahrq.gov/ehc/products/151/392/Health\%2 0Literacy\%20Protocol\%20\%282-9-2010\%29.pdf

4. Sørensen K, Van den Broucke S, Fullam J, et al. Health literacy and public health: a systematic review and integration of definitions and models. BMC Publ Health 2012;12:1-13.

5. Peerson A, Saunders M. Health literacy revisited: what do we mean and why does it matter? Health Promot Int 2009;24:285-96.

6. DeWalt DA, Berkman ND, Sheridan S, et al. Literacy and health outcomes. A systematic review of the literature. J Gen Intern Med 2004;19:1228-39.

7. Nutbeam D. Health literacy as a public health goal: a challenge for contemporary health education and communication strategies into the 21st century. Health Promot Int 2000;15:259-67.

8. Chew LD, Griffin JM, Partin MR, et al. Validation of screening questions for limited health literacy in a large VA outpatient population. J Gen Intern Med 2008;23:561-6.

9. Ishikawa H, Nomura K, Sato M, et al. Developing a measure of communicative and critical health literacy: a pilot study of Japanese 
office workers. Health Promot Int 2008;23:269-74.

10. Van der Vaart R, Drossaert C, Taal E. Validation of the Dutch functional, communicative and critical health literacy scales. Patient Educ Couns 2012;89:82-8.

11. Wallace LS, Rogers ES, Roskos SE. Screening items to identify patients with limited health literacy skills. J Gen Intern Med 2006;21:874-7.

12. Schulz P, Nakamoto K. Health literacy and patient empowerment in health communication: the importance of separating conjoined twins. Patient Educ Couns 2013;90:4-11.

13. Moreno-Londoño AM, Schulz PJ. judgment skills, a missing component in health literacy: development and validation of a tool for asthma patients in the italian speaking region of Switzerland. Arch Public Health 2014;72:12.

14. Nathan RA, Sorkness CA, Kosinski M, et al. Development of the asthma control test: a survey for assessing asthma control. J Allergy Clin Immunol 2004;113:59-65.

15. Laforest L, Hasnaoui AEI, Pribil C, et al. Asthma patients' selfreported behaviours toward inhaled corticosteroids. Res Med 2009;103:1366-75.

16. The Global Initiative for Asthma (GINA). Dissemination and implementation of asthma guidelines. Report 2002. Available from: http://www.ginasthma.org/pdf/GINADissemination.pdf

17. Gibson PG, Powell H, Wilson A, et al. Self-management education and regular practitioner review for adults with asthma. Cochrane Database Syst Rev 2002;3:CD001117.

18. Thai AL, George M. The effects of health literacy on asthma selfmanagement. J Asthma Allergy 2010;1:50-5.

19. Denford S, Campbell JL, Frost J, Greaves CJ. Processes of change in an asthma self-care intervention. Qual Health Res 2013;23:141929.

20. Tattersfield AE. Limitations of current treatment. Lancet 1997;350S:24-7.

21. Thoonen BPA, Schermer TRJ, van den Boom G, et al. Self-management of asthma in general practice, asthma control and quality of life: a randomised controlled trial. Thorax 2003;58:30-6.
22. Braman SS. The global burden of asthma. Chest 2006;130:4S-12S.

23. Dowson CA, Town GI, Frampton C, Mulder RT. Psychopathology and illness beliefs influence COPD self-management. J Psychosom Res 2004;56:333-40.

24. Kolbe J, Vamos M, Fergusson W, et al. Differential influence on asthma self-management knowledge and self-management behavior in acute severe asthma. Chest 1996;110:1463-8.

25. Becker MH. Theoretical models of adherence and strategies for improving adherence. In: Schumaker SA, Schron EB, Ockene JK, eds. The handbook of health behavior change. New York: Springer Publishing; 1990: pp. 5-43.

26. Bernard-Bonnin AC, Stachenko S, Bonin D, et al. Self-management teaching programs and morbidity of pediatric asthma: a metaanalysis. J Allergy Clin Immunol 1995;95:34-41.

27. Sin DD, Man J, Sharpe H, et al. Pharmacological management to reduce exacerbations in adults with asthma. A systematic review and meta-analysis. JAMA 2004;292:367-76.

28. Paasche-Orlow MK, Riekert KA, Bilderback A, et al. Tailored education may reduce health literacy disparities in asthma self-management. Am J Respir Crit Care Med 2005;172:980-6.

29. Cochrane GM, Horne R, Chanez P. Compliance in asthma. Respir Med 1999;93:763-9.

30. Buston KM, Wood SF. Non-compliance amongst adolescents with asthma: listening to what they tell us about self-management. Fam Pract 2000;17:134-8.

31. Bender BG, Long A, Parasuraman B, Tran ZV. Factors influencing patient decisions about the use of asthma controller medication. Ann Allergy Asthma Immunol 2007;98:322-8.

32. Chapman KR, Walker L, Cluley S, Fabbri L. Improving patient compliance with asthma therapy. Respir Med 2000;94:2-9.

33. van der Mortel TF. Faking it: social desirability response bias in self-report research. Aust J Adv Nurs 2008;25:40-8.

34. MacCallum RC, Zhang S, Preacher KJ, Rucker DD. On the practice of dichotomization of quantitative variables. Psychol Methods 2002;7:19-40. 